Christine Hikel, Nicole Kramer, Elisabeth Zellmer

\title{
Impulse für eine neue Frauen- Politikgeschichte
}

Im 20. Jahrhundert bestimmten politische Systemwechsel, Kriege und Krisen, aber auch Konsolidierung und Demokratisierung das Verhältnis des Staats zu seinen Bürgerinnen neu. Hatte die Mehrheit der Männer, nicht zuletzt wegen der allgemeinen Wehrpflicht, die Aufwertung zum Staatsbürger bereits im 19. Jahrhundert erreicht, zogen die Frauen erst 1918 mit der Einführung des Frauenwahlrechts nach. Dies war jedoch mehr Markstein als Schlussstein eines Politisierungsprozesses ${ }^{1}$, der in den folgenden Jahrzehnten in unterschiedlichen Ausprägungen seine Fortsetzung fand. Totalitäre Herrschaft, die nationale Vereinnahmung im Zweiten Weltkrieg, Demokratisierung oder die politische Planungs- und Steuerungseuphorie der 1960er Jahre verschoben die Grenze zwischen dem, was als "politisch", und dem, was als "privat" galt. Frauen gerieten in den Fokus des Staats und erhielten neue Mōglichkeiten der politischen Teilhabe. Davon profitierten nicht nur die Eliten, sondern die Frauen insgesamt, aiso auch das sprichwortliche "Lieschen Müller“2.

Lieschen Müller entwickelte sich im 20. Jahrhundert zum Synonym für die deutsche Durchschnittsfrau. Erstmals tauchte diese Figur aber bereits im 18. Jahrhundert auf - zusammen mit dem Soldaten Fritzchen stand sie für die Untertanen des Königs Friedrich Wilhelm I., der - so die landläufige Sicht - als sparsamer Regent

\footnotetext{
1 Vgl. Konrad H. Jarausch/Michael Geyer, Zerbrochener Spiegel. Deutsche Geschichte im 20. Jahrhundert, München 2005, S. 278-302.

2 Zur "Genealogie" und „Karriere" Lieschen Müllers vgl.: Artikel „Liese", in: Grimm's deutsches Wörterbuch, Bd.6, Leipzig 1885; Artikel "Lieschen Müller", in: Hermann Paul, Deutsches Wörterbuch, 5.völlig neu bearb. u. erw. Aufl. von Werner Betz, Tübingen 1966; Christina von Hodenberg, Konsens und Krise. Eine Geschichte der westdeutschen Medienöffentlichkeit 1945-1973, Göttingen 2006, S.80; Anja Kruke, Demoskopie in der Bundesrepublik Deutschland. Meinungsforschung, Parteien und Medien 1949-1990, Düsseldorf 2007; Lieschen Müller, Ottos Verwandte, in: taz vom 1.2.2003; Alexander Mitscherlich, Cocteaus Witwe. Zur Genealogie von Lieschen Müller, in: ders., Gesammelte Schriften VI. Politisch-publizistische Aufsätze 1, hrsg. von Herbert Wiegandt, Frankfurt a.M. 1983, S. 278-282; Spiegel-Verlag/Hausmitteilung, in: Der Spiegel vom 28.10.1968, S. 5.
} 
sein Volk arm und ungebildet lieB. Als Sinnbild der gemeinen Preußin genoss sie einen zweifelhaften Ruf: unauffällig, gewöhnlich, bisweilen gar dumm. Nach dem Zweiten Weltkrieg änderte sich das Bild: Die neu entstehende Disziplin der Meinungsforschung griff Figuren wie Lieschen Müller oder Otto Normalverbraucher auf und erklärte sie zum Pendant der einfachen Frauen und Mānner auf der Straße. Da insbesondere die Parteien daran interessiert waren, was die schweigende Mehrheit dachte, gewann Lieschen Müller eine politische Dimension. Aus dem einfältigen Geschöpf wurde eine Staatsbürgerin, deren Spuren man aber nur selten in Parteien, Parlamenten oder anderen Institutionen findet. Doch dass sie vorhanden sind - gerade auch außerhalb dieser etablierten Arenen -, wird dieser Band zeigen. Die Vielfalt weiblichen Engagements, die der Blick auf Lieschen Müller offen legt, spiegelt sich in den einzelnen Beiträgen wider, die sich konzeptionell an drei Leitlinien orientieren:

1. Geschlecht: Der akteurszentrierte Ansatz soll keine Rückkehr zur Frauengeschichte der 1970er Jahre sein, der es vor allem darum ging, Frauen als Subjekte in der Geschichte sichtbar zu machen. Die einzelnen Beiträge beziehen Geschlecht als konstituierenden Faktor der Vergesellschaftung in ihre Untersuchungen mit ein und tragen somit den methodischen Debatten der Geschlechtergeschichte Rechnung. Die Zuschreibungen, die "die Frauen“ pauschal zu einem monolithischen Block innerhalb der Gesellschaft machen, sind nur eine Seite der sozialen Praxis. Wie Frauen diese Rollenmuster wahrnahmen, wie sie sich dazu verhielten, ob sie sie ignorierten oder als verbindlich ansahen, zeigt sich erst, wenn die Akteurinnen selbst im Zentrum der Untersuchung stehen. Es soll also nicht um den Blick des Staats auf die Frauen, sondern um deren Haltung und Verhalten dem Staat gegenüber gehen. Frauen strebten nach Integration, ließen sich in Dienst nehmen, stellten Forderungen, verlangten Mitsprache, setzten Grenzen oder liefen dagegen Sturm.

2. Staat: Das 20. Jahrhundert gilt als „Zeitalter der Extreme“ (Eric Hobsbawm). Vor allem Deutschland pendelte zwischen Demokratie und Diktatur. Mit den Systemwechseln gingen politisch-normative und gesellschaftliche Verãnderungen einher, die die Spielräume der Mitgestaltung von Frauen im Staat neu bestimmten. Dabei ist es wichtig festzuhalten, dass die Geschichte weiblicher Partizipation keine bruchlose, lineare Erfolgsgeschichte ist, die nach und nach ein Mehr an politischer Teilhabe und Mitbestimmung erbracht hat. Vielmehr lassen sich Zeitfenster finden, in denen das Engagement von Frauen besonders hervortrat, während es in anderen 
Perioden in den Hintergrund rückte. Die Gründe dafür, dass sich solche Zeitfenster offneten, sind vor allem zwei Faktoren geschuldet: Zum einen der Absicht des Staats, Frauen zu mobilisieren und zur Unterstützung des herrschenden politischen Systems zu gewinnen, zum anderen der in bestimmten Phasen besonders stark empfundenen Überzeugung, die Lage von Frauen sei defizitär und die weibliche Bevölkerung im oder durch den Staat nicht ausreichend reprāsentiert. Die zeitliche Lāngsschnittperspektive ermöglicht es, Brüche und gegensãtzliche Entwicklungen zu untersuchen, die deutschen Diktaturen in die Analyse zu integrieren und schließlich eine Basis für den diachronen Vergleich zu legen. Dabei wird unter anderem erkennbar, dass sich die Zweite Frauenbewegung ganz selbstverständlich auf die Erste berief. Dennoch unterschieden sich deren Aktivistinnen in ihrer Staatsauffassung grundlegend von ihren politischen Großmüttern. Ging es in der ersten Hālfte des 20. Jahrhunderts um die Anerkennung als Staatsbürgerinnen und die Verpflichtung gegenüber dem Gemeinwesen, stritten westdeutsche Frauen mehr als 50 Jahre spãter im Kontext einer stabilen Demokratie und auf der Basis gesicherter Grundrechte für mehr Selbstbestimmung und gegen staatliche Eingriffe.

3. Partizipation: Da der Untersuchungszeitraum das gesamte 20. Jahrhundert umfasst, ist es notwendig, den Partizipationsbegriff von seiner traditionellen demokratietheoretischen Konnotation zu lösen. Danach richtet sich Partizipation auf das politische System, in dem Bürger politische Entscheidungen "freiwillig“, „individuell" oder „im Verbund mit anderen“ und „zu ihren Gunsten“ beeinflussen können. In Definitionen wie dieser klingt ein normatives Verstāndnis von politischer Beteiligung an, die über die Interessenvertretung hinaus auf demokratische Ideale wie "Selbstverwirklichung" und "Teilhabe in möglichst vielen Bereichen der Gesellschaft" verweist ${ }^{3}$. Allerdings, so ist dagegen einzuwenden, strebt jede Form politischer Herrschaft nach Legitimitât und Akzeptanz, für die ein gewisses $\mathrm{Ma} \beta$ an Beteiligung der Bevölkerung unabdingbar ist. Auch diktatorische und autoritäre Regime versuchen, mit ihren politischen Botschaften Zustimmung zu gewinnen. Gerade in totalitären Systemen ist es ein Charakteristikum, dass der Grad der politischen Durchdringung und Mobilisierung der gesamten Bevölkerung ausgesprochen hoch ist. Der entscheidende Unterschied im Vergleich zu Demokratien liegt im Grad der Offenheit

${ }^{3}$ So z.B. unter dem Stichwort "Partizipation" in: Lexikon der Politik. Bd. 7: Politische Begriffe, hrsg. von Dieter Nohlen/Rainer-Olaf Schultze/Suzanne S. Schüttenmeyer, München 1998, S.470-472, hier S. 470. 
der politischen Sphäre, die in Diktaturen - vor allem durch Gewalt und Repression - stark vorstrukturiert ist und kaum Platz für Pluralismus lāsst. Partizipation verlāuft hier innerhalb enger Grenzen. Teilhabe und Mitwirkung spielten sich in den Diktaturen des 20. Jahrhunderts nicht auf der Ebene politischer Entscheidungsfindung ab, vielmehr konnte die Mehrheit der Bevōlkerung lediglich an der Schnittstelle zwischen politischen Vorgaben und deren Umsetzung Einfluss nehmen. Hier eröffneten sich die Handlungsspielräume für die „kleinen“ Mānner, aber auch für die „kleinen“ Frauen, die man bisher zumeist übersehen hat. Ein entsprechend erweiterter Partizipationsbegriff ${ }^{4}$ ermōglicht es, demokratische und diktatorische Systeme gleichermaßen in den Blick zu nehmen. Partizipation kann so zu einer Analyse- und Vergleichskategorie für das gesamte 20. Jahrhundert werden.

Die vorliegenden Beiträge erschließen über die Frage nach der politischen Teilhabe von Frauen exemplarisch die deutsche Geschichte des 20. Jahrhunderts. Auf der Grundlage jüngst abgeschlossener oder noch laufender empirischer Studien vermessen sie ein vernachlāssigtes Forschungsfeld anhand innovativer Fragestellungen und geben Impulse für eine neue Frauen-Politikgeschichte, die sowohl Frauen als Akteurinnen als auch die Bedingungen ihres Handelns in herrschafts-, sozial- und erfahrungsgeschichtlicher Perspektive untersucht. Der Band gliedert sich in fünf Abschnitte. Den Anfang macht ein Überblick über die Geschichte von Frauen als Staatsbürgerinnen in Demokratie und Diktatur. Durch den Blick auf das gesamte 20. Jahrhundert werden die Zeitfenster sichtbar, in denen die Teilhabe der weiblichen Bevölkerung verstärkt thematisiert, begünstigt, eingefordert, aber auch wieder zurückgedrängt wurde.

Ein erstes Fenster öffnete sich in den Jahren nach dem Ersten Weltkrieg. Der Systemwechsel von 1918 veränderte die Rahmenbedingungen weiblicher Teilhabe radikal. Der neue Status als vollwertige Staatsbürgerinnen hob die Partizipation von Frauen im Staat auf eine neue Stufe. Es ging aber nicht nur um Rechte und Möglichkeiten, eigene Interessen durchzusetzen und Gesellschaft und Politik mitzugestalten. Partizipation hieB nicht zuletzt Dienst für das Volk und Mitarbeit am Gemeinwesen.

4 Die Erweiterung von Politik- und Partizipationsbegriffen hat bereits die Neue Politikgeschichte angeregt. Vgl. v.a. Ute Frevert, Neue Politikgeschichte. Konzepte und Herausforderungen, in: dies./Heinz-Gerhard Haupt (Hrsg.), Neue Politikgeschichte. Perspektiven einer historischen Politikforschung, Frankfurt a.M. 2005, S. 9-26. 
Der Nationalsozialismus konnte die politische Integration von Frauen nicht gãnzlich revidieren. Der Notwendigkeit, die Zustimmung der weiblichen Bevölkerung zu gewinnen, konnte sich auch das Dritte Reich nicht verschließen. Allerdings galt dies lãngst nicht für alle; Frauen, die aus rassischen und weltanschaulichen Gründen stigmatisiert wurden, mussten zwischen 1933 und 1945 erfahren, wie ihnen die staatliche Macht jegliche Partizipationsmöglichkeit versagte.

Für die „Volksgenossinnen“ gestaltete sich die Situation hingegen anders: Sie sollten in den totalen Staat und die nationalsozialistische „Volksgemeinschaft" integriert werden, und viele nahmen dieses Inklusionsangebot bereitwillig auf. Das Dritte Reich erwies sich dabei auf der Ebene der symbolischen Repräsentation als männlich dominiert, während die politische und soziale Praxis - wenn auch in hierarchischer Abstufung - auf der Integration und Mitwirkung beider Geschlechter beruhte. Dies war das Einfallstor für die Gestaltungsmacht von Frauen im nationalsozialistischen Männerstaat.

Mit dem Ende des Zweiten Weltkriegs verloren nationalsozialistische Politikvorstellungen ihre Bedeutung. An deren Stelle traten die westalliierten Forderungen nach Demokratisierung, die zumindest in Teilen der deutschen Bevölkerung auf bereits vorhandene Einsichten stießen. Die weibliche Mitbestimmung in der neuen Demokratie sollte kein Elitenphänomen sein, sondern möglichst allen offenstehen. Partizipation hatte verschiedene Ansatzpunkte und unterschiedliche Formen. Frauenvereine und -verbände, die zum Teil an ihre Arbeit aus der Zeit vor 1933 anknüpfen konnten, spielten hãufig eine wichtige Rolle bei der Politisierung der weiblichen Bevōlkerung und etablierten sich als Vorfeldorganisationen. Impulse kamen zudem von der "großen Politik“, deren Steuerungs- und Deutungshoheit bis in den privaten Bereich ihrer Bürger hineinreichte. Die staatlichen Institutionen wurden von diesen jedoch auch gefordert, nicht zuletzt von Frauen, die sich in materiellen Notlagen befanden.

Wesentlich konfliktreicher gestaltete sich das Verhältnis der Zweiten Frauenbewegung zum demokratischen Staat, die in den 1970 er Jahren entstand. "Das Private ist politisch" war einer ihrer zentralen Slogans, doch die Aktivistinnen wehrten sich zugleich gegen Eingriffe von oben und betrachteten staatliche Steuerungsversuche mit Skepsis. Sie nutzten die Öffentlichkeit, um etablierte Institutionen herauszufordern und um diesen ihre Grenzen aufzuzeigen. Als Teil der sozialen Bewegungen der 1960er und 1970er Jahre formulierte die Neue Frauenbewegung in der Bundesrepublik 
Protest und sorgte für Polarisierung. Mit Einschrānkungen galt dies auch für die DDR, wo nichtstaatliche Frauengruppen in den 1980er Jahren zu Trägern einer Gegenkultur wurden.

Der vierte Band der Reihe "Zeitgeschichte im Gespräch“ ist das Ergebnis eines Workshops über die politische Partizipation von Frauen im 20. Jahrhundert, der im Oktober 2007 in München stattfand. Wegbereiter dieses Forschungsinteresses war das Institut für Zeitgeschichte München-Berlin. Die Tagung und der Sammelband haben sich nur durch die Unterstützung und Mitarbeit so vieler verwirklichen lassen. Wir danken dem Direktor des Hauses, Horst Möller, seinem Stellvertreter Udo Wengst und der Verwaltungsleiterin Ingrid Morgen, die dieses Projekt möglich gemacht haben. Stellvertretend für die vielen Kolleginnen und Kollegen möchten wir Ingrid Baass, Ute Elbracht, Alexander Markus Klotz, Edith Raim, Anne Rohstock, Thomas Schlemmer und Hans Woller nennen, die unsere Ideen und unseren Eifer stets unterstützt und uns den Rücken freigehalten haben. Unser Dank gilt nicht zuletzt den Autorinnen und Autoren der Beiträge für die gute Zusammenarbeit. 